\title{
Intelligent Beaconing Principle based Congestion Free Routing in VANET
}

\author{
Sankaragomathi.M', Srigayathri.M², Vijaya Lakshmi. ${ }^{3}$, Senthil Madasamy.N ${ }^{4}$ \\ UG Student, Department of Information Technology, Kamaraj College of Engineering and Technology, \\ Virudhunagar, Tamil Nadu, India ${ }^{1,2,3}$ \\ Assistant Professor, Department of Information Technology, Kamaraj College of Engineering and Technology, \\ Virudhunagar, Tamil Nadu, India ${ }^{4}$
}

\begin{abstract}
Beacons are an application which relays the details, such as distance between two nodes, coverage area and the node positions. A Beaconing activity is helpful for estimating the complete status of node and identify whether the node is suitable to make the communication or not. Once if the Beaconing activity is analyzed, the traffic estimation principles take place to avoid congestions ${ }^{[7]}$ over a network. Vehicular networks ${ }^{[8]}$ play an important role in wireless communications, which allows the node to move from one location to another location dynamically without any interruption. In this system wireless environment is created with the number of nodes and allow the source and destination nodes able to move from one location to another location dynamically during data transmission. In the proposed approach Fair Adaptive Beaconing Rate for Inter-vehicular Communications (FABRIC) algorithm ${ }^{[4]}$ is used, which solves the problem of Network Utility Maximization (NUM) in a wireless environment. For all, the entire simulation results prove that the proposed approach is more convenient to perform wireless data transmission, without any congestion or traffic breakages.
\end{abstract}

Keywords: VANET, Beaconing activity, Vehicular Networks, Congestion.

\section{INTRODUCTION}

Vehicular Ad-Hoc Network, or VANET, a technology that VANET is a type of MANET and also it provides the uses cars as nodes in a network to create a mobile following which is clearly shown in Figure 1.1

network. VANET turns every participating car into a • Vehicle-to-vehicle communication

wireless router or node, allowing cars approximately 100 - Vehicle-to-Roadside communication

to 300 meters of each other to connect and which in turn, - Routing based communication ${ }^{[10]}$

create a network with a wide range of network. As cars fall out of the signal range and when they drop out of the network, other moving cars can join in, connecting vehicles to one with another so that a mobile Internet is created. It is estimated and calculated that the first systems $^{[12]}$ that will integrate this technology and used in real time are police and fire vehicles to communicate with each one another for safety purposes. VANET promises us to provide safer roads, assures less or no accidents. It also provides more efficient driving by letting the driver know about the traffic ${ }^{[11]}$ while travelling.

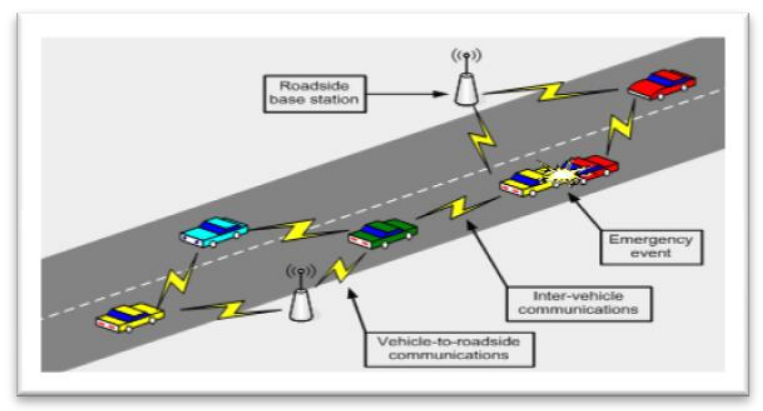

Figure 1.1
Vehicle-to-Vehicle Communications, The inter vehicle communication configuration uses multi hop multicast/broadcast to transmit the packet information ${ }^{[5]}$ over multiple hops to a group of receivers.

Vehicle-to-Roadside Communications, The vehicle to roadside communication configuration indicates a single hop broadcast where the roadside items will send a broadcast message to all equipped vehicles within the surroundings.

Routing based Communications, The routing based communication $^{[10]}$ is a multi hop unicast, where the messages are propagated in a multi hop fashion until the vehicle carrying the desired data is reached to the destination.

To secure the VANET environment, first we have to discover who are the attackers attacking the network, their nature, and capacity to damage the system ${ }^{[12]}$. On the basis of capacity of the network these attackers may be of three types.

Insider and Outsider, Insiders are the authenticated members of the network.Outsiders are the intruders and hence they have limited capacity to attack. 
Malicious and Rational, Malicious attackers have not any personal benefit to attack the nodes; they just harm the functionality of the network without any personal intention. Rational attackers have the personal profit, hence they are easily predictable.

Active and Passive, Active attackers generate signals or packet to transmit whereas passive attackers only sense the nodes of the network. An active attack is a network damage in which a hacker attempts to make changes to data on the target or data travelling route to the target. An active attack, in computing security, is an attack characterized by the attacker attempting to break into the system $^{[12]}$ and enter into the system. During an active attack, the intruder will introduce data into the system as well as potentially change data within the system ${ }^{[12]}$ and collapse. A passive attack is a network attack in which a system is monitored and sometimes scanned for open ports and vulnerabilities. The purpose is solely to gain information $^{[5]}$ about the target node and no data is changed on the target. A passive attack, in computing security, is an attack characterized by the attacker listening in on communications. In those attacks, the intruder/hacker does not attempt to break into the system ${ }^{[12]}$ or otherwise change data.

VANET is an application form of MANET but it has its own distinct characteristics as follows:

High Mobility, The nodes in VANETs usually are moving at very high speed. This makes harder to identify a node's position and making protection to the node.

Rapidly changing network topology, Due to high node mobility and because of the random speed of vehicles, the position of a node changes often. As a result of this, network topology in VANETs will change frequently.

Unbounded network size, VANET can be implemented for one city or several cities or throughout the countries. This means that network size in VANET is unbounded geographically.

Frequent exchange of information ${ }^{[5]}$, the ad hoc nature of VANET inspires the nodes to gather information from the other nearby vehicles and roadside units. Hence the information exchange among node becomes often.

Time Critical, the information in VANET must be delivered to the nodes within the time period so that a decision can be made by the node and perform action accordingly.

\section{RELATED WORK}

A.Distributed and Fair Beaconing Rate Adaptation for Congestion Control in Vehicular Networks ${ }^{[2]}$

Esteban Egea-Lopez et al proposed an overview of the Beaconing activity which was achieved to indicate the congestion $^{[9]}$ free communication more efficiently. The positive and active nodes are found clearly. The node to node communication was also clearly established. It requires limited signaling overhead between vehicles.Cooperative inter-vehicular ${ }^{[8]}$ applications depend on the exchange of broadcast single-hop status messages between vehicles, called beacons. The load on the wireless channel $^{[15]}$ due to periodic beacons can prevent the transmission of other types of messages, what leads to channel congestion ${ }^{[7]}$ due to beaconing activity. The problem of controlling the beaconing rate ${ }^{[1]}$ on each vehicle by modeling it as a Network Utility Maximization (NUM) problem. The NUM methodology allocates a rigorous framework to design a broad family of simple and decentralized algorithms ${ }^{[4]}$ which proved convergence guarantees to a fair allocation solution. The main objective is beaconing rate control and propose the Fair Adaptive Beaconing Rate for Intervehicular ${ }^{[8]}$ Communications (FABRIC) algorithm ${ }^{[4]}$. This uses a particular algorithm to solve the NUM problem. The desired fairness notion in the allocation can be established with the parameters included in the algorithm ${ }^{[4]}$. The procedure is robust against errors such as packet losses due to fading, collisions or hiddennode interferences.

B. Analysis of Information Dissemination in Vehicular ${ }^{[2]}$ Ad-Hoc Networks with Application to Cooperative Vehicle Safety Systems

Ching-Ling Huang et al proposed the dissemination of tracking information ${ }^{[5]}$. The main advantages include channel occupancy that quantifies the success of information and these findings are used to design feedback control schemes. But logical justifications are not yet met.Cooperative vehicle safety systems ${ }^{[12]}$ (CVSSs) depend on vehicular ${ }^{[2]}$ ad-hoc networks (VANETs) for the correct delivery of critical vehicle tracking information. ${ }^{[5]}$ The wireless channel ${ }^{[15]}$ in such systems is shared by vehicles within the transmission range of one another. Due to the near-linear spatial distribution of vehicles in a highway scenario, the vehicular network ${ }^{[2]}$ is heavily affected by the hidden node interference, which has limited capacity. The performance of the vehicle tracking application that is the basis for CVSS is therefore significantly affected by the performance of the underlying network. The two main parameters that affected the network condition and performance are the range and rate (frequency) of transmission of safety and tracking messages. They analyzed the effect of different choices of rate and range and they also presented the models of network performance that quantifies in terms of its ability to disseminates tracking information. ${ }^{[5]}$ This is followed by a thorough analysis of the hidden node affected VANET that show channel ${ }^{[15]}$ occupancy or busy ratio can be used as a feedback measure that quantifies the success of disseminated information ${ }^{[5]}$ and the CVSS, under different conditions of network design. These findings are used to design feedback control schemes for transmission range adaptation, which are robust to variations of road and network traffic. ${ }^{[11]}$

C. LIMERIC : A Linear Adaptive Message Rate Algorithm for DSRC Congestion Control

Charles et al proposedThe vehicle-to-vehicle (V2V) and vehicle-to infrastructure (V2I) communication has great 
promise for significantly reducing the human and financial costs of vehicle collisions. A common feature of this communication was the broadcasting the device state information ${ }^{[5]}$ at regular intervals (e.g., vehicle speed and location or traffic ${ }^{[11]}$ signal state and timing). or controlled, the consolidate of these broadcasts will congesting the channel under the traffic scenarios, reducing the effectiveness of collision avoidance applications that use transmitted information ${ }^{[5]}$. The Active congestion ${ }^{[7]}$ control using the techniques is a topic of great interest for establishing the scalability of this technology. They defined a new adaptive congestion control algorithm ${ }^{[4]}$ which is applied to the devices of some message rate ${ }^{[1]}$ in this vehicular ${ }^{[2]}$ environment. While comparing with other published approaches they depend on binary control, the LInear MEssage Rate ${ }^{[1]}$ Integrated Control (LIMERIC) algorithm ${ }^{[4]}$ took advantage of full-precision control inputs that are available on the wireless channel. The result was a provableconvergence to fair and efficient channel utilization in that environment, under simple setting adaptive parameters. This "perfect" convergence avoids the limit cycle behavior that is inherent to binary control. They also discussed several practical associated with implementing LIMERIC, including guidelines for the choice of the system ${ }^{[13]}$ parameters to obtain desired utilization outcomes, then saturation technique that maintained robust convergence under all conditions, convergence with asynchronous updates, and used channel load to determine the aggregated message rate ${ }^{[1]}$ that is observable at a receiver. This paper also extended the analysis and it has the two classes, the first one is measurement noise in the input signal and delay in the updated process.

D. Vehicular Networking: A Survey and Tutorial on Requirements, Architectures, Challenges, Standards and Solutions

Boangoat Jarupan et al proposed vehicular networking ${ }^{[2]}$ has potential to enable diverse applications cooperative the traffic ${ }^{[11]}$ safety, traffic efficiency and infotainment. In that tutorial paper they said that characteristics of inter vehicular networks ${ }^{[2]}$, these are provide an applications and associated requirements, along with challenges and their proposed solutions. In addition, they provide the current and past major ITS programs and projects in the USA, Japan and Europe. Moreover, vehicular networking ${ }^{[2]}$ architectures and protocol suites employed in such programs and projects in USA, Japan and Europe are discussed

E. Trust management for Vehicular Networks: An Adversary-Oriented

Carlos et al proposed Cooperative Intelligent Transportation Systems (C-ITS), represented by vehicular ad-hoc networks ${ }^{[2]}$ (VANETs), are the key components which are mainly contributing to the Smart City and Smart World paradigms. Based ${ }^{[10]}$ on the exchange that are continuous of both periodic and event triggered messages, smart vehicles can enhance safety of the road, while also providing support for comfort applications. In addition to the different communication protocols which are securing such communications and establishing a certain trustiness among vehicles are the main challenges to be considered, since the presence of dishonest peers can lead to unwanted situations.Up to this end, existing security solutions are typically divided into two main categories, cryptography and trust, where trust appeared to cryptography on some specific adversary models and environments where the latter was not enough to mitigate all possible attacks.

\section{III . EXISTING SYSTEM}

In past system several location estimation and channel management algorithms ${ }^{[4]}$ are proposed, but most of them are able to bring the channel load to the desired level, none of them is able to meet all the network requirements and all of them consider a very basic approach to fair allocation $^{[16]}$ of beaconing rates, without a formal definition and rigorous convergence support. In some cases, the combination of a basic notion and its particular implementation may result in an unnecessarily low rate ${ }^{[1]}$ and so a decrease in safety as we discuss later with an example. There are two questions related to this issue. One is which the appropriate notion of fairness in vehicular networks ${ }^{[2]}$ is and whether different scenarios require different notions of fairness. Another one is how to enforce a particular fairness notion and control it dynamically ${ }^{[6]}$.

\section{PROPOSED SYSTEM}

A. Introduction

In the proposed approach, we proposed modified Fair Adaptive Beaconing Rate ${ }^{[1]}$ for Inter-vehicular Communications (FABRIC) algorithm with Network Utility Maximization principles, which allows us to design a broad family of decentralized and simple algorithms, with proved convergence guarantees to a fair allocation solution $^{[16]}$, supported by the rigorous developments of NUM theory. In addition to that Beaconing principle is achieved to indicate the congestion ${ }^{[7]}$ free communication more efficiently and propose a particular algorithm and validate it with extensive simulations in static and dynamic $^{[6]}$ scenarios.

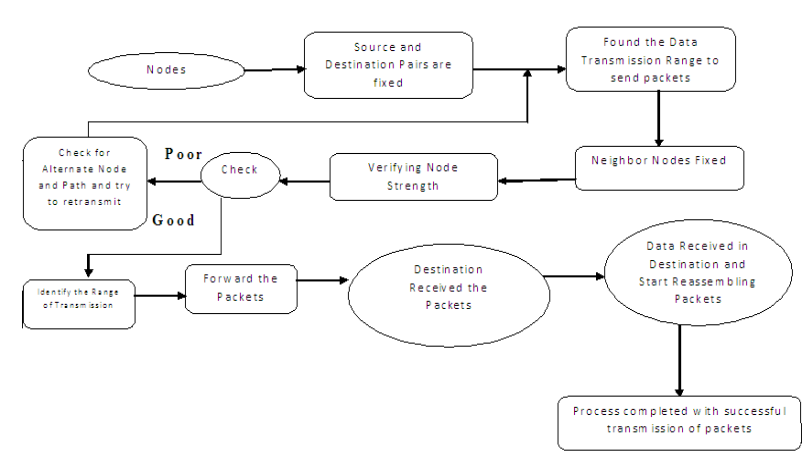

Figure 4.1 System archircture 
We have modified the FABRIC algorithm and calculated the nearest neighbor and transmitted only to those neighbours to reach the destination.

\section{Modules Description}

\section{WIRELESS COMMUNICATION MODEL}

Wireless Communication Model, VANET environment is designed with number of node establishments in dynamic $^{[6]}$ nature. This module provides the provision to create $\mathrm{n}$ number of nodes dynamically over the wireless environment. The dynamic ${ }^{[6]}$ wireless network in ns 2 can be modeled using rand function in the Tool Command Language (TCL) script. The number of nodes in the network is dynamic. These networks allocate the dynamic location for each node. The nodes are fixed in the program. Dynamically created nodes are configuredwithspecific parameters of a mobile wireless node. After creating the nam file and trace file, we set up topography object.

\section{Beaconing module}

The Beaconing Module is used to maintain the details, such as the distance between two nodes, coverage area and the node positions. A Beaconing activity is helpful for estimating the complete status of node and identify whether the node is suitable to make the communication or not. Only after identifying that a node is active it is fixed to involve in the communication process. If it is found to be dead, then in such case the participating node will search for some other node which best suits for the communication. Anothermajor factor that has to be included while checking for the routing path is the coverage area. It simply means that when a node is selected for communication,then it should be in that particular coverage area.

\section{Congestion free communication module}

The congestion ${ }^{[7]}$ free communication module is achieved with the help of Fair Adaptive Beaconing Rate ${ }^{[1]}$ for Intervehicular Communications (FABRIC) algorithm, ${ }^{[4]}$ which uses a particular scaled gradient projection algorithm to solve the dual of the Network Utility Maximization (NUM) problem. The main advantage with this algorithm $^{[4]}$ is that to find the routing path the node itself involve in this routing algorithm. The participating node itself checks for the required items for communication.

\section{Graphical evaluations}

A graphical evaluation module shows a clear perspective to the users, which shows the end-to-end delay management mechanisms, packet delivery ratio estimations, lifetime improvement principles in a graphical way.

\section{EXPERIMENAL RESULTS}

We give our simulation results and compared the performance of our system with the existing system. For simulation we used Network simulator. After setting up the platform, software named ns2 was set upon it which was used for all the analysis and simulation work apart from other tools used. Ns2 is the de facto standard for network simulation. Its behavior is highly trusted within the networking community. We have compared and verified that our system gives a better result compared to the existing system ${ }^{[13]}$

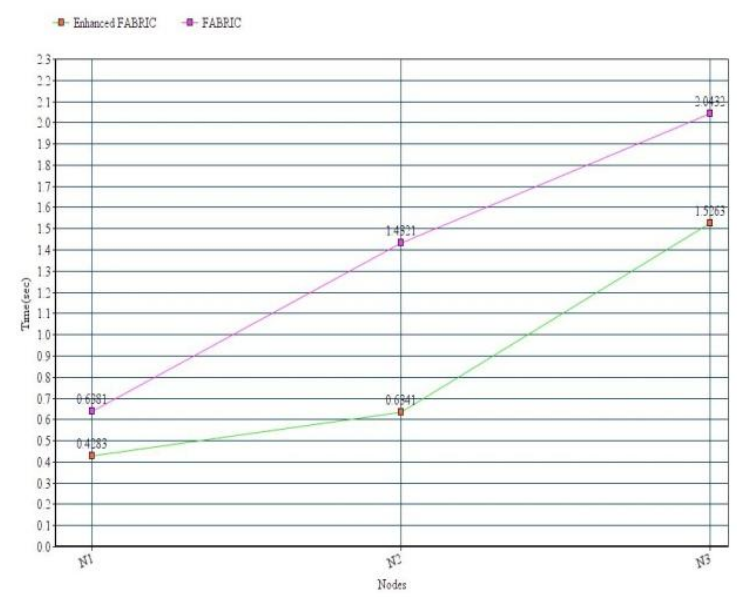

Figure 5.1Performance Evaluation.

\begin{tabular}{|c|c|c|}
\hline Nodes & $\begin{array}{c}\text { Time(Seconds) } \\
\text { FABRIC }\end{array}$ & $\begin{array}{c}\text { Time(Seconds) } \\
\text { Enhanced FABRIC }\end{array}$ \\
\hline N1 & 0.4283 & 0.6381 \\
\hline N2 & 0.6341 & 1.4321 \\
\hline N3 & 1.5263 & 2.0432 \\
\hline
\end{tabular}

We have compared our result considering the nodes and their time on which they reach their neighbor node which is of shortest distance.

\section{CONCLUSION AND FUTURE ENHANCEMENT}

In this system we model for the first time, to the best of our knowledge, the problem of beaconing rate control in vehicular networks as a NUM rate allocation problem. This modeling opens the door to formally define and apply fairness notions to beaconing rate allocations in vehicles. In addition, it provides a mathematical framework to develop decentralized and simple algorithms with proven convergence guarantees to a fair allocation solution.

In this respect, we have presented a family of algorithms based on the gradient optimization of the dual of the rate allocation problem. Within this family, we have proposed the Fair Adaptive Beaconing Rate for Intervehicular Communications algorithm. FABRIC, is a decentralized rate allocation algorithm with theoretical and empirical convergence properties, which requires limited signaling overhead between vehicles.

We have validated FABRIC by exhaustive simulations in both static and dynamic scenarios, for different position distributions and propagation models. Results show that 
FABRIC effectively generates fair beaconing rate allocations. Moreover, only in a few steps, the algorithm is able to move the rates out of the congestion state and close to the optimal allocation. Simulations also confirm that the algorithm is robust against packet losses due to collisions or fading.

Our results have been compared with LIMERIC+PULSAR, a relevant rate allocation scheme in vehicular networks. There are still a number of practical considerations and implementation alternatives that can be evaluated in order to tune the algorithm. First, the $b$ parameter controls the convergence speed and the amplitude of fluctuations and there is a wide range of possible values meeting the convergence condition to test. Second, filtering of unreliable links may provide a more accurate measurement of congestion (gradient computation) in fading scenarios. Even the use of alternative congestion measurements such as the measured CBT can be tested. We intend to carry out an extensive evaluation of these matters in a future work.

Additionally, from a more general perspective, we have shown how different values of the fairness parameter a result, in different allocations, which may be more adequate depending on the intended application or scenario. As we discussed with a particular example, a too basic fairness notion may directly influence the safety of the vehicles. Therefore, it is necessary to study, which is the suitable notion of fairness in vehicular networks and with whether different scenarios require different notions of fairness or not. This is an open question left as future work but a positive answer implies that it is also a necessary mechanism to dynamically control fairness. In this sense, one of the key advantages of FABRIC and our approach is that the fairness allocations can be controlled with this single parameter.

Moreover, this approach allows to use different values for each vehicle or even to use totally different utility functions, which can be both dynamically changed. And vehicles do not need to know the functions or values used by other vehicles. Therefore, in addition to the practical utility of our proposal, in our opinion, one of the main contributions of this system is the establishment of the NUM model as an effective and rich framework for developing beaconing rate control schemes in vehicular networks. Consequently, as a future work, we intend to further explore variations of the discussed problem in the context of vehicular networks.

In particular, a comparative application and evaluation of alternative fairness notions and the introduction of heterogeneous utility functions and constraints in the problem.

Finally, our model also provides support for the quality of service needs of the applications, which usually require to control additional variables such as transmit power. In fact, many recent proposal let the application freely set minimum values for one or severals variables and then apply a distributed control for the rest of them over the remaining capacity, which might results in violations of the MBL. It is interesting to have a more integrated approach and so we are working on a reformulation of the problem to jointly control power and beaconing rate.

\section{REFERENCES}

[1] B. Kim, I. Kang, and H. Kim, "Resolving the unfairness of distributed rate control in the IEEE WAVE safety messaging," IEEE Trans. Veh. Technol., vol. 63, no. 5, pp. 2284-2297, Jun. 2014.

[2] Boangoat Jarupan , Eylem Ekici, Geert Heijenk, Georgios Karagiannis, Onur Altintas Kenneth Lin and Timothy Weil March 2014, Vehicular Networking: A Survey and Tutorial on Requirements, Architectures, Challenges, Standards and Solutions , VOL 40

[3] Carlos T. Calafatey, Juan-Carlos Canoy, Nasreddine Lagraa Chaker Abdelaziz Kerrache, Pietro Manzoniy JUNE 2015, Trust management for Vehicular Networks: An Adversary-Oriented Overview, , VOL 50

[4] Charles,E,Rohrs, Gaurav Bansal, John B,Kenney, NO. 9,NOVEMBER 2013, LIMERIC : A Linear Adaptive Message Rate Algorithm for DSRC Congestion Control, VOL. 62

[5] Ching-Ling Huang, Hariharan Krishnan, Raja Sengupta, Yaser,P,Fallah, JANUARY 2014 , Analysis of Information Dissemination in Vehicular Ad-Hoc Networks with Application to Cooperative Vehicle SafetySystems, VOL. 60, NO.1

[6] ControlMing-Yang Chen, Student, Wonjong Rhee, IEEE,Mehdi Mohseni, and John M. Cioffi, Distributed Crosstalk Management for Upstream VDSL Using Dynamic Power VOL. 60, NO. 4, APRIL 2012

[7] E. Egea-Lopez, J. J. Alcaraz, J. Vales-Alonso, A Festag, and J. Garcia-Haro, "Statistical beaconing congestion control for vehicular networks," IEEE Trans. Veh. Technol., vol. 62, no. 9, pp. 41624181, Nov. 2013.

[8] Hanlin Meng, Kan Zheng, Lei Lei, Lu Hou, Ning Lu and Qiang Zheng, July/August 2016, Soft-Defined Heterogeneous Vehicular Network: Architecture and Challenges

[9] J. B. Kenney, G. Bansal, and C. E. Rohrs, "LIMERIC: A linear adaptive message rate algorithm ${ }^{[4]}$ for DSRC congestion control," IEEE Trans. Veh. Technol., vol. 62, no 9, pp. 4182-4197, Nov. 2013.

[10] J. Mo and J. Walrand, "Fair end-to-end window-based congestion control," IEEE/ACM Trans. Netw., vol. 8, no. 5, pp. 556-567, Oct. 2000.

[11] Kelly, Frank, "Charging and rate control for elastic traffic," Eur. Trans. Telecommun., vol. 8.1, pp. 33-37, 1997.

[12] Marc Torrent-Moreno, Jens Mittag, Paolo Santi, and Hannes Hartenstein,Vehicle-to-Vehicle Communication: Fair Transmit Power Control for Safety-Critical Information, IEEE VOL. 58, NO. 7, SEPTEMBER 2009

[13] M. Sepulcre, J. Mittag, P. Santi, H. Hartenstein, and J. Gozalvez, "Congestion and awareness control in cooperative vehicular systems," Proc. IEEE, vol. 99, no. 7, pp. 1260-1279, Jul. 2011.

[14] Omid Ardakanian, Srinivasan Keshav, and Catherine Rosenberg Real-Time Distributed Control for Smart Electric Vehicle Chargers: From a Static to a Dynamic Study, VOL. 5, NO. 5, SEPTEMBER 2014

[15] Sreejith Sreekumar, Bikash Kumar Dey, and Sibi Raj B. Pillai Distributed Rate Adaptation and Power Control in Fading Multiple Access channel, VOL. 61, NO. 10, OCTOBER 2015

[16] ShahbazPanahi, and Min Dong, Achievable RateRegionunder Joint DistributedBeamforming andPowerAllocationforTwo-WayRelay NetworksShahram VOL. 11, NO. 11, NOVEMBER 2012

[17] Y. P. Fallah, C. L. Huang, R. Sengupta, and H. Krishnan, "Analysis of information dissemination in vehicular Ad-Hoc networks with application to cooperative vehicle safety systems," IEEE Trans. Veh. Technol., vol. 60, no 1, pp. 233-247, Jan. 2011. 


\section{BIOGRAPHIES}

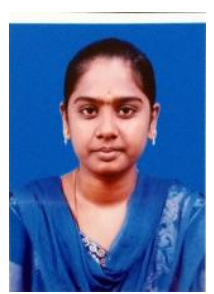

M. Sankaragomathi - Pursuing Final year of Bachelor of Technology in Information Technology from Anna University Chennai in Kamaraj College of Engineering and Technology, Virudhunagar. Her research interest is networking.

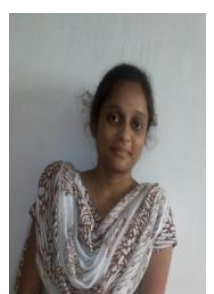

M.Srigayathri - Pursuing Final year of Bachelor of Technology in Information Technology from Anna University Chennai in Kamaraj College of Engineering and Technology, Virudhunagar. Her research interest is networking.

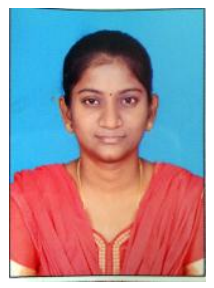

P.Vijaya Lakshmi - Pursuing Final year of Bachelor of Technology in Information Technology from Anna University Chennai in Kamaraj College of Engineering and Technology, Virudhunagar. Her research interest is networking.

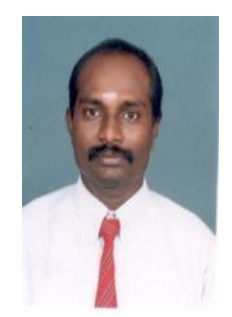

N.Senthil Madasamy - Received Bachelor of Engineering in Computer Science and Engineering from Manonmaniam Sundaranar University in Government college of engineering ,Tirunelveli in 1998 and Master of engineering in Computer Science and Engineering from Anna University, Chennai in 2007. Currently he is doing Ph.D. in Peer to Peer Network at Anna University, Chennai. He is working an Assistant Professor in Kamaraj College of Engineering and Technology, TamilNadu, India. His research interests include Networking, Parallel Computing and Image Processing. 